\title{
A Rare Case of Primary Mucocutaneous Histoplasmosis Presenting as a Non-Healing and Disfiguring Upper Lip Ulcer.
}

\author{
D Muchuweti ${ }^{1}$, R Makunike-Mutasa ${ }^{1}$, E Muguti, G Nyandoro' ${ }^{2}$. \\ ${ }^{1}$ Department of Surgery, \\ ${ }^{2}$ Department of Community Medicine,College of Health Sciences, University of \\ Zimbabwe, Box AP 178, Avondale, Harare, Zimbabwe
}

Correspondence to: George Nyandoro Email: georgenyandoro@yahoo.com https://dx.doi.org/10.4314/ecajs.v22i1.14

BA 27-year-old female patient presented with a non-healing disfiguring upper lip ulcer for 10 months. At the time of presentation $50 \%$ of her lip had been eroded. She had had several consultations and had been on several antibiotic courses without improvement. Histopathological evaluation of an incisional biopsy specimen revealed that she had histoplasmosis. This prompted an HIV test, as this disease is common in immunocompromised patients. Once the diagnosis was clinched she was commenced on Itraconazole with complete resolution of the ulcer. Antiretroviral therapy was also initiated.

Key words: HIV, Histoplasmosis, Histoplasma Capsulatum, mucocutaneous, ulcer, immunocompromised, Itraconazole

\section{Introduction:}

Non-healing ulcers can be disfiguring especially when they are located on the face. Besides the distress of disfigurement the patient has additional stress of worrying about malignancy especially squamous cell carcinoma. Infectious aetiological agents need to be excluded in chronic ulcers. Histoplasmosis is a granulomatous infectious mycosis caused generally, but not exclusively by Histoplasma Capsulatum. Samuel Darling first described the disease in 1905. Commonly it has pulmonary manifestations but can affect reticuloendothelial organs ${ }^{1}$. Oral lesions manifesting primarily are rare ${ }^{2}$. They tend to occur in conjunction with disseminated histoplasmosis especially in HIV-infected patients ${ }^{3}$. A histological examination of an incisional biopsy secures the diagnosis of histoplasmosis and should prompt an HIV test. Medical treatment is all that is needed in these chronic ulcers due to Histoplasma Capsulatum. There is prompt response to Itraconazole. We present a case of primary oral histoplasmosis in a patient who as a result of the diagnosis was subsequently found to be HIV positive. Response to Itraconazole was dramatic, much to the relief of the patient.

\section{Case Report}

A 27-year-old female patient was referred to our outpatient surgical clinic with a 10-month history of a non-healing ulcer on the upper lip. She had been on several antibiotic courses 
some based on pus swab microscopy, sensitivity and culture with no improvement. She had no co-morbid conditions and her HIV status was unknown at the time of presentation.

On physical examination she was a young female patient who was distressed about the ulcer. Her blood pressure, pulse and temperature were normal. She had mobile cervical and axillary lymphadenopathy. Her Cardiovascular and Respiratory examination were normal, as were the rest of the systems. There was an irregularly- shaped ulcer involving more than $50 \%$ of her upper lip. The edges were punched out with no attempt at healing. Necrotic tissue was evident on the most superior part of the ulcer. The pre-treatment image of the ulcer is shown in Figure 1.

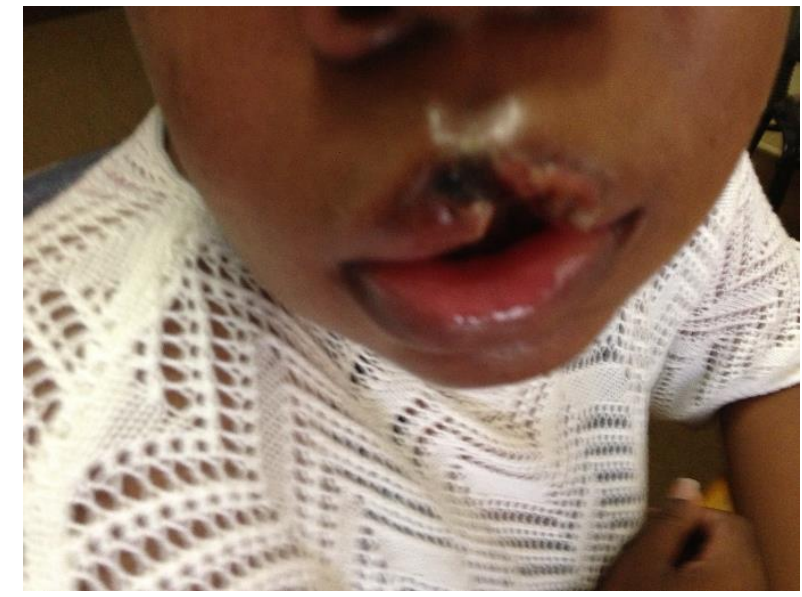

Fig 1: Pre-treatment image of the ulcer

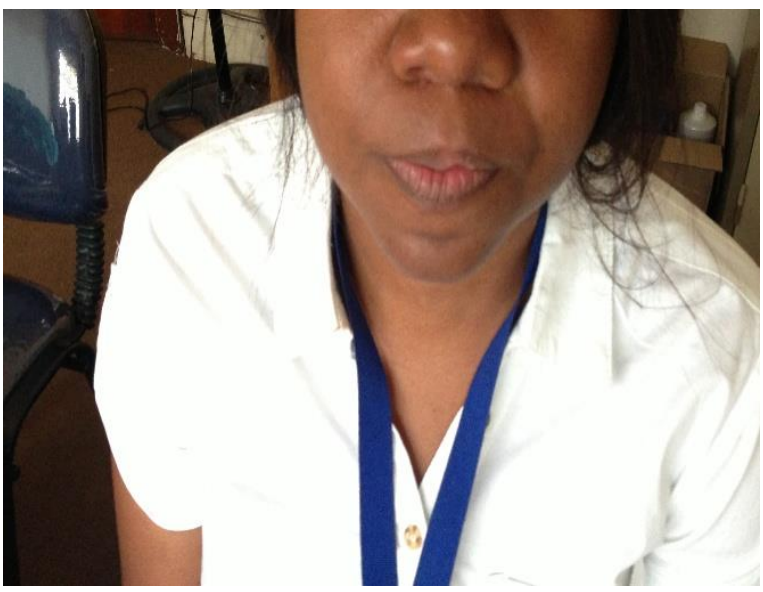

Fig 3: Post treatment image of the upper lip.

A diagnosis of a chronic mucocutaneous ulcer of the upper lip was made. Hematological investigations showed a low white cell count of $3.36 \times 10^{9} / \mathrm{l}$ and low hemoglobin of $11.20 \mathrm{~g} / \mathrm{dl}$. Her biochemistry results were normal. An incisional biopsy was done in theatre. Microscopic examination showed some poorly formed granulomas with multinucleated giant cells ingesting fungal spores. Appearances were consistent with Histoplasmosis and there was no evidence of malignancy. The patient was commenced on high dose itraconazole for 6 weeks. The results were dramatic. There was complete healing of the ulcer with no need for plastic reconstructive surgery. Post treatment images of the upper lip are shown in Figure 2. After appropriate counseling an HIV test was done. Her HIV test was positive with a CD4 cell count of 250 cells $/ \mathrm{ml}$. She was commenced on anti-retroviral therapy.

\section{Discussion}

Histoplasmosis is a fungal disease caused by Histoplasmas Capsulatum. Samuel Darling first described the disease in 1905. Histoplasma Capsulatum is a dimorphic fungus found in warm humid environment that contains bird and bat excreta ${ }^{1}$. Sources of infection are birds, bats and soil contaminated with their droppings ${ }^{2}$.Spores are infective to humans and small animals. The disease is transmitted by the inhalation of airborne conidia. Introduction of the spores through the skin and mucous membrane will result in infection in most cases. 


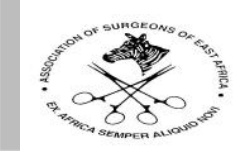

Histoplasma Capsulatum causes acute and/or chronic pulmonary disease but can affect other reticuloendothelial organs ${ }^{3}$. Oral lesions manifesting primarily are rare ${ }^{4}$. They tend to occur in conjunction with disseminated histoplasmosis especially in HIV-infected patients 5 . Recently primary mucocutaneous ulcers have been reported with greater frequency in HIVpositive patients ${ }^{6}$. This was the case with our patient who was later found to be HIV positive. Differential diagnoses include lichen planus, squamous cell carcinoma and tuberculous ulcer.

Diagnosis is established by identifying the small intracellular yeast cells $(2-5 \mathrm{~mm})$ of Histoplasma in smears of mucocutaneous lesions, sputum, peripheral blood, and bone marrow or biopsy specimens. Our patient had diagnosis established from a biopsy specimen because squamous cell carcinoma was one of the differential diagnoses. Histopathological identification of Histoplasma Capsulatum in the sections provides conclusive evidence of the disease ${ }^{7}$.

In immunocompetent patients the disease is self-limiting. Pulmonary histoplasmosis in HIV patients is managed with Amphotercin B at a dose of $2 \mathrm{gms}$ intravenous for 10 weeks.

Mucocutaneous or oral lesions are best treated with Intraconazole at a dose of 200mg twice a day for 4 weeks $^{3}$. Itraconazole has a rapid action and prevents relapses ${ }^{8}$. Our patient had a rapid recovery.

\section{Conclusion}

In conclusion patients presenting with oral ulcers should be meticulously examined and laboratory investigations should be done to exclude histoplasmosis. Biopsies of chronic ulcers must always be done early to avoid ineffective treatment, which contributes to the delay of the diagnosis, as was the case with our patient and also in averting unnecessary surgery, which may cause further disfigurement. Histoplasmosis being an opportunistic infection may be the first and only indication of HIV infection ever with CD4 cell counts below 400, as was the case in this patient. This case highlights the importance of tissue diagnosis in the management of non-healing ulcers and in heralding possible HIV positive cases.

\section{References:}

1. Wheat J, Sarosi G, Mckinsey D, Hamill R, Johnson $P$, et al. Practice guidelinesfor the management of patients with histoplasmosis. Clin Infect Dis. 2000;30:688-95.(Pub Med)

2. Rippon JW. The pathogenic fungi and the pathogenic actinomycetes. $3^{\text {rd }}$ ed Saunders: Philadelphia; 1988. p 381-417

3. Hay RJ, MooreMK. Mycology. In: Tony B, Stephen B, Neil C, Christopher G, editors. Rooks Text Book of Dermatology. Blackwell Science Limited: London; 2004. P.88-90

4. Cohen PR. Oral histoplasmosis in HIV-infected patients. Oral Surg Oral Med Oral Pathol. 1994; 78:277-8(Pub Med)

5. Sayal SK, Prasad PS, Sanghi S. Disseminated histoplasmosis: Cutaneous presentation. Indian J Dermatol Venereol Leprol 2003; 69:90-1 
6. Iqbal, Firoz C, Hedley G, Schifter M. Oral Histoplasmosis: A Rare Cause of Chronic Nonhealing ulcer in an Immunocompetent Individual. 2010, vol 42-Issue-pS15.

7. Wheat JL. Current diagnoses of histoplasmosis. Trends in microbiology. 2003;11:488-94. (Pub Med)

8. Economopoulou P, Laskaris G, Kittas C. Oral histoplasmosis as an indicator of HIV infection. Oral Surg Oral Med Oral Pathol Oral Radiol Endod. 1998;86:203-6 